\title{
Abdominal mass in a patient with Crohn's disease
}

\author{
D Ravichandran, CD Johnson
}

A 28-year-old man with a 10-year history of Crohn's disease presented with four-week history of a gradually enlarging right lower abdominal mass. Two months previously he had suffered a relapse of his disease with right iliac fossa pain and was commenced on mesalazine. A barium follow-through at that time showed active Crohn's disease of the terminal ileum with a stricture.

On admission he was clinically well and afebrile but had a $10 \times 10 \mathrm{~cm}$ mildly tender lump in the right iliac fossa. His haemoglobin was $10.8 \mathrm{~g} / \mathrm{dl}$, white cell count $14.7 \times 10^{9} / 1$ with neutrophilia, serum albumin $26 \mathrm{~g} / \mathrm{l}$, C-reactive protein $249 \mathrm{mg} / \mathrm{l}$ and erythrocyte sedimentation rate 96 . He was treated with high-dose intravenous steroids and antibiotics but the mass continued to increase in size (figure 1). Computed tomography (CT) was performed (figure 2).

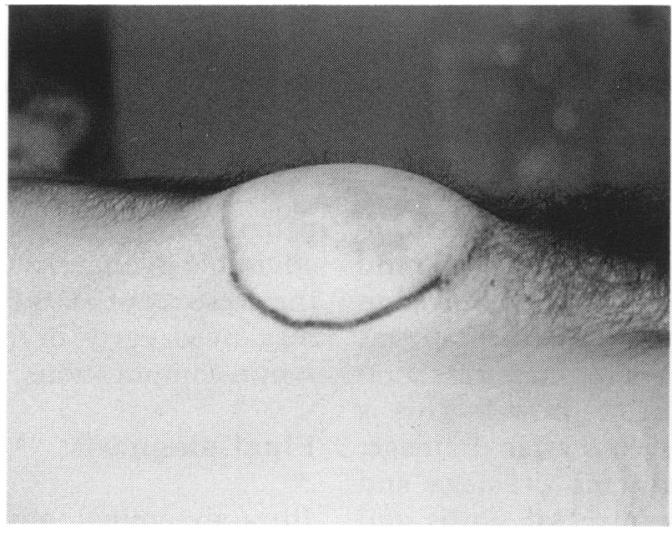

Figure 1 Photograph showing the abdominal mass in the right iliac fossa

Southampton

D Ravichandran

CD Johnson

\section{Correspondence to}

Mr CD Johnson, Senior

Lecturer in Surgery,

University Surgical Unit, F Level, Centre Block,

Southampton General

Hospital, Tremona Road,

Southampton SO166YD, UK

Accepted 2 March 1995

\section{Questions}

1 List three possible differential diagnoses

2 What is the radiological diagnosis?

3 How common is this complication in Crohn's disease?

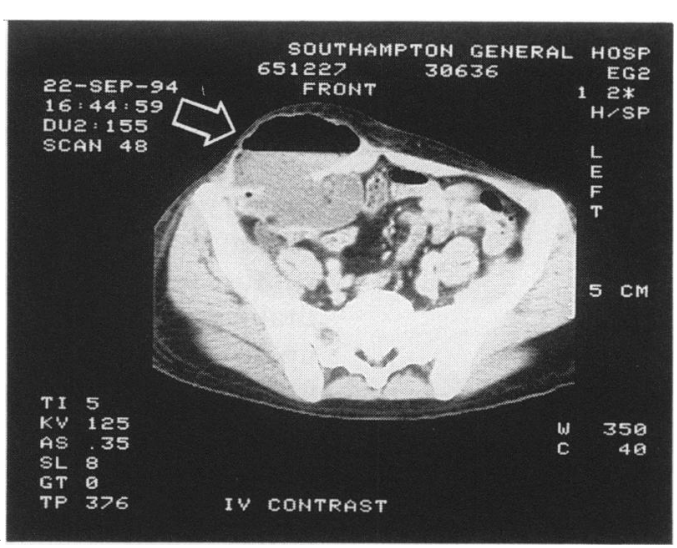

Figure 2 CT of the mass seen in figure 1, showing a large intra-abdominal abscess in the right iliac fossa communicating with a more superficial component lying just underneath the skin

4 What immediate treatment is indicated and what is the likely outcome?

5 Is the initial treatment with intravenous steroids and antibiotics justifiable? 


\section{Answers}

\section{QUESTION 1}

- intra-abdominal abscess

- carcinoma of the bowel - ileocaecal region (incidence of colonic cancer in Crohn's is about 3-5\%)

- mass of inflamed bowel loops

- other incidental causes

\section{QUESTION 2}

There is a large intra-abdominal abscess in the right iliac fossa which is continuous with a large subcutaneous component containing air via a communication through the abdominal wall this has the appearance of the 'collar stud abscess' described in tuberculosis. The subcutaneous component in one area is covered only by thinned skin (arrow) and is ready to rupture spontaneously.

\section{QUESTION 3}

Intra-abdominal abscess is a common complication of Crohn's disease with a reported incidence of $12-30 \% .^{1}$ Intra-peritoneal abscess (eg, interloop, subphrenic, mesenteric, and hepatic) is commoner than retroperitoneal (eg, psoas) or pelvic abscess.

\section{QUESTION 4}

As the abscess is almost pointing and about to rupture spontaneously, immediate incision and drainage is indicated. This will be followed in up to $85 \%$ of cases $^{2}$ by an enterocutaneous fistula which almost always requires resection of the affected segment of the bowel. This is usually performed 6-8 weeks after drainage. The interval between abscess drainage and bowel resection allows control of sepsis and correction of metabolic and nutritional deficits which are often present in these patients, thus creating optimal circumstances for elective

1 Cybulsky IJ, Tam P. Intra abdominal abscess in Crohn's disease. Am Surg 1990; 56: 678-82.

Steinberg DM, Cooke WT, Alexander-Williams J. Abscess and fistulae in Crohn's disease. Gut 1973; 14: 865-9.

3 Voeller G, Britt L. Surgical management of perforated Crohn's disease. Am Surg 1990; 56: 100-3.

4 Felder JB, Adler DJ, Korelitz BI. The safety of corticosteroid therapy in Crohn's disease with an abdominal mass. Am $f$ Gastroenterol 1991; 86: 1450-5. bowel resection later. However, some advocate a one-stage procedure involving abscess drainage and bowel resection and anastomosis. ${ }^{3}$ In this patient drainage was followed by an enterocutaneous fistula which was treated by the resection of the affected ileocolic segment of the bowel.

\section{QUESTION 5}

Corticosteroid therapy has traditionally been considered to be unsafe in Crohn's disease patients with an abdominal mass for fear that this will impair immunity, mask symptoms of inflammation, and cause dissemination of infection. There is, however, evidence that steroid therapy, when combined with intravenous antibiotics, is safe in carefully chosen patients. Even small abscesses may respond to this treatment and the need for subsequent bowel resection may be avoided. ${ }^{4}$ This could be attempted whenever feasible as it is always useful to avoid surgery in Crohn's disease, not only because of the high risk of recurrent disease but also because patients who have had bowel resections for 'perforating' complications such as fistula and abscess are likely to require another bowel resection, with its associated morbidity and mortality, twice as quickly as those who have not. ${ }^{5}$

If it is nevertheless decided to treat these patients with such a regimen, it would seem advisable to obtain a CT scan early, to exclude the presence of a large or difficult abscess which may need early drainage to prevent serious septic complications.

\section{Final diagnosis}

Intra-abdominal abscess in a patient with Crohn's disease

Keywords: Crohn's disease, abdominal abscess

5 Greenstein AJ, Lachman P, Sachar DB, et al. Perforating and non perforating indications for repeated operations in Crohn's disease. Evidence of two clinical forms. Gut 1988; 29: 588-92. 